\title{
Using a sledgehammer to crack a nut: The burdensome appropriate use criteria program
}

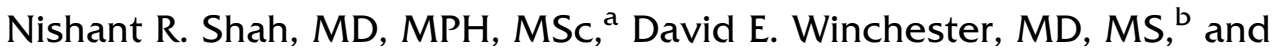 \\ Andrew $M$. Freeman, $M D^{c}$ \\ a Division of Cardiology, Department of Medicine, Brown University Alpert Medical School, \\ Providence, RI \\ b Cardiology Section, Malcom Randall VAMC, Department of Medicine, University of Florida \\ College of Medicine, Gainesville, FL \\ c Division of Cardiology, Department of Medicine, National Jewish Health, Denver, CO
}

Received Nov 22, 2019; accepted Nov 25, 2019

doi: $10.1007 / \mathrm{s} 12350-019-01978-4$

\section{See related article, pp. 1988-1997}

The 2013 Multimodality Appropriate Use Criteria (AUC) for the Detection and Risk Assessment of Stable Ischemic Heart Disease define clinical scenarios where cardiac testing is unlikely to improve patients' medical care and is therefore 'rarely appropriate'. 1 Recent meta-analyses suggest that up to 1 in 5 cardiac tests performed can be designated rarely appropriate ${ }^{2,3}$, similar to broader assessments suggesting that 20-30\% of all advanced diagnostic imaging tests are unnecessary. ${ }^{4}$ In response, the United States Congress passed the Protecting Access to Medicare Act of 2014, which directed the Centers for Medicare and Medicaid Services (CMS) to establish the AUC Program. The program, set to begin in an 'education and operations testing period' on January 1, 2020, will universally require clinicians to document consultation of AUC-based clinical decision support mechanisms (CDSMs) on every Medicare claim related to advanced diagnostic imaging tests.

In the present issue of JNC, Divakaran et al describe their findings after piloting a homegrown, point-of-care CDSM to aid clinicians in cardiac test selection for inpatients. Their 12-question CDSM focused on ascertaining patient history, symptoms, primary indication for testing, pre-test probability of obstructive coronary

Reprint requests: Andrew M. Freeman, MD, Division of Cardiology, Department of Medicine, National Jewish Health, 1400 Jackson St.

J317, Denver, CO 80206, USA; andrew@docandrew.com

J Nucl Cardiol 2021;28:1998-2000.

1071-3581/\$34.00

Copyright (C) 2019 American Society of Nuclear Cardiology. artery disease (CAD), and ability to exercise. In retrospective analysis of 288 inpatient cardiac tests ordered with this CDSM, Divakaran et al found that $99 \%$ were appropriate or maybe appropriate despite $30 \%$ discordance between two adjudicating physicians regarding the specific AUC indication under which the test was performed. Divakaran et al also found that information contained within the electronic health record was sufficient to assess the appropriateness of only $87 \%$ of tests and that ability to exercise was the most valuable additive information provided by the CDSM. Together, these critical pre-implementation data bolster increasing concerns among front-line providers and healthcare facilities $^{5}$ that the AUC Program is akin to using a sledgehammer to crack a nut and that significant modifications to the program are likely needed.

\section{THE AUC PROGRAM INDISCRIMINATELY IMPOSES FINANCIAL AND ADDITIONAL WORKLOAD BURDEN ON HEALTHCARE SYSTEMS WITHOUT ACCOUNTING FOR THE QUALITY OF CURRENT PRACTICE PATTERNS}

The finding of Divakaran et al that only $1 \%$ of the inpatient cardiac tests they reviewed were rarely appropriate parallels a prior study from another large tertiary care center that only $2 \%$ of cardiac tests done to evaluate inpatients with chest pain or other anginal-equivalent symptoms were rarely appropriate. ${ }^{6}$ The term 'rarely appropriate' was chosen to convey the concept that an otherwise seemingly unnecessary test may actually be relevant in selected patients. While the medical community has not agreed on an acceptable rate of rarely appropriate tests, few would argue that $1-2 \%$ is 
excessive. Given this low rate of rarely appropriate inpatient cardiac testing, perhaps such tests should be excluded completely from the scope of the AUC Program. Now, it is reasonable to argue that practice patterns at large tertiary care centers may not reflect those at smaller centers where rates of rarely appropriate inpatient cardiac testing may be higher. However, even if that were true, all centers (regardless of size) should have the opportunity to demonstrate the baseline quality of their practice patterns before being forced to invest significant time and resources to develop the human and computing infrastructure needed to comply with the AUC Program. Furthermore, in an era of increasing metrics, increased provider burnout (often due to lack of power or perceived autonomy), and decreasing reimbursements, the value of adding administrative 'hoops' like the AUC program should be shared with healthcare providers and health systems a priori with pilot data and that simply has not been done.

\section{INHERENT SUBJECTIVITY IN THE INTERPRETATION OF CLINICAL SCENARIOS STRONGLY SUGGESTS THAT THE AUC PROGRAM WILL HAVE LIMITED IMPACT}

As the old adage says: if you want two different opinions, ask two different physicians. Divakaran et al found that two physicians adjudicating the specific AUC indication under which cardiac tests were performed were discordant $30 \%$ of the time, often due to differing assessments of patients' pre-test probability of obstructive CAD. In their discussion, the authors astutely conclude that this is evidence of significant subjectivity in the interpretation of clinical scenarios when applying AUC. Indeed, categorical assessment of patients' pretest probability of having obstructive CAD as low, intermediate, or high is explicitly described in the 2012 Guideline for the Diagnosis and Management of Patients with Stable Ischemic Heart Disease as 'somewhat arbitrary" $^{7}$ and has only become more so amidst an ongoing 'battle of the scores ${ }^{\prime} 8$ including the updated Diamond-Forrester model ${ }^{9}$ and the CAD Consortium models. ${ }^{10}$ Both of these scores are heavily influenced by classification of patients' symptoms as non-specific chest pain, atypical angina, or typical angina which is independently highly subjective and often based on missing or incomplete clinical documentation. Furthermore, there is little consensus on how patients solely with dyspnea on exertion fit into this classification. Some documents suggest this symptom should be grouped with 'noncardiac chest pain'; however, at many institutions, dyspnea is treated as an anginal equivalent in everyday practice. In addition to the subjectivity associated with assessment of pre-test probabilities, application of the Multimodality AUC are also dependent on clinicians' subjective assessment of patients' ability to exercise adequately during cardiac stress testing. Finally, and perhaps most concerning, recent data have shown that application of different AUC to a specific patient and test can result in substantial discordance in assessments of appropriateness. ${ }^{11}$ Given all this subjectivity and the fact that clinicians can 'shop' through CDSMs (Table 1) to find appropriate indications for tests, it is unlikely that clinicians will purposefully order a test rated as rarely appropriate by a CDSM. From that perspective, the AUC Program and its associated CDSMs will immediately 'solve' the problem of rarely appropriate cardiac testing on paper but they won't have any meaningful impact in improving the cardiovascular care that patients receive.

\section{THE AUC PROGRAM SHOULD NOT MOVE FORWARD UNTIL ASSOCIATED CDSMS ARE BETTER INTEGRATED INTO ELECTRONIC HEALTH RECORDS}

Like the CDSM designed by Divakaran et al, most commercially available CDSMs approved for use under the AUC Program require clinicians to manually enter data that is already discretely stored in the electronic health record (i.e., history of CAD, presence of cardiovascular risk factors, current medications, weight) to determine the specific AUC indication under test appropriateness will be assessed. This not only burdens clinicians with unnecessary and duplicative administrative work, it is also subject to provider errors in data retrieval and entry. As Divakaran et al found in their analyses, clinicians often miss critical information during real-time assessment of patients and their records (i.e., history of coronary revascularization, prior cardiac testing, current smoking status) that can significantly alter the specific AUC indication they believe a test is being performed under. Until CDSMs can automatically pull relevant data to assist clinicians-and verify their input-policies like the AUC Program that mandate CDSM use should be optional. In the current era of machine learning, artificial intelligence, and advanced algorithmic computing, most of the key clinical variables should be able to readily discerned and preapproved in nearly all cases. Further, it may make sense to consider spending resources on developing such technology in place of a provider-carried, burdensome 'consult the AUC' paradigm. For the time being, in lieu of CDSMs, it may be reasonable to use more local solutions that leverage personalized audit and feedback to reduce the prevalence of rarely appropriate cardiac testing. $^{12}$ 
Table 1. Clinical decision support mechanisms qualified by CMS for use under the appropriate use criteria program as of June 2019

\section{Qualified CDSMs}

AgileMD's Clinical Decision Support Mechanism

AIM Specialty Health ProviderPortal ${ }^{a}$

Applied Pathways CURION ${ }^{\mathrm{TM}}$ Platform

Cranberry Peak ezCDS

eviCore healthcare's Clinical Decision Support

Mechanism

EvidenceCare's Imaging Advisor

Inveni-QA's Semantic Answers in Medicine

MedCurrent OrderWise

Medicalis Clinical Decision Support Mechanism

National Decision Support Company CareSelect ${ }^{a}$

National Imaging Associates RadMD

Reliant Medical Group CDSM

Sage Health Management Solutions Inc. RadWise

Stanson Health's Stanson CDS

Test Appropriate CDSM ${ }^{\mathrm{a}}$

${ }^{\mathrm{a}}$ Free tool available

To conclude, it is clear that rarely appropriate cardiac testing occurs far more frequently than it should and should be eliminated where possible. The AUC program and its associated CDSMs, while well-intentioned, needlessly burden clinicians and healthcare systems with significant effort and costs while providing a relatively meaningless 'fix.' Local solutions that emphasize personalized feedback to ordering clinicians and are not tied to reimbursement may be better-suited to achieve the desired improvements in patients' care. Alternatively, making technology work for patients and clinicians in the ways described above could simplify and revolutionize workflows, and that's where the real 'sledgehammer' is needed.

\section{Disclosures}

Dr. Winchester is supported by VA HSR\&D Career Development Award 13-023. The views expressed here are those of the authors only.

\section{References}

1. Wolk MJ, Bailey SR, Doherty JU, et al. ACCF/AHA/ASE/ASNC/ HFSA/HRS/SCAI/SCCT/SCMR/STS 2013 multimodality appropriate use criteria for the detection and risk assessment of stable ischemic heart disease: A report of the American College of Cardiology Foundation Appropriate Use Criteria Task Force, American Heart Association, American Society of Echocardiography, American Society of Nuclear Cardiology, Heart Failure Society of America, Heart Rhythm Society, Society for Cardiovascular Angiography and Interventions, Society of Cardiovascular Computed Tomography, Society for Cardiovascular Magnetic Resonance, and Society of Thoracic Surgeons. J Am Coll Cardiol 2014;63:380-406.

2. Ladapo JA, Blecker S, O’Donnell M, Jumkhawala SA, Douglas PS. Appropriate use of cardiac stress testing with imaging: A systematic review and meta-analysis. PLoS ONE. 2016;11(8):e0161153.

3. Elgendy IY, Mahmoud A, Shuster JJ, Doukky R, Winchester DE. Outcomes after inappropriate nuclear myocardial perfusion imaging: A meta-analysis. J Nucl Cardiol 2016;23:680-9.

4. Lehnert BE, Bree RL. Analysis of appropriateness of outpatient CT and MRI referred from primary care clinics at an academic medical center: how critical is the need for improved decision support? J Am Coll Radiol 2010;7:192-7.

5. Doukky R. PAMA implementation: The road ahead. J Nucl Cardiol. 2019. https://doi.org/10.1007/s12350-019-01942-2.

6. Gertz ZM, O’Donnell W, Raina A, Litwack AJ, Balderston JR, Goldberg LR. Application of appropriate use criteria to cardiac stress testing in the hospital setting: Limitations of the criteria and areas for improved practice. Clin Cardiol 2015;38:8-12.

7. Fihn SD, Blankenship JC, Alexander KP, et al. 2014 ACC/AHA/ AATS/PCNA/SCAI/STS focused update of the guideline for the diagnosis and management of patients with stable ischemic heart disease: A report of the American College of Cardiology/American Heart Association Task Force on Practice Guidelines, and the American Association for Thoracic Surgery, Preventive Cardiovascular Nurses Association, Society for Cardiovascular Angiography and Interventions, and Society of Thoracic Surgeons. Circulation 2014;130:1749-67.

8. Di Carli MF, Gupta A. Estimating pre-test probability of coronary artery disease: Battle of the scores in an evolving CAD landscape. JACC Cardiovasc Imaging 2019;12:1401-4.

9. Genders TS, Steyerberg EW, Alkadhi H, et al. A clinical prediction rule for the diagnosis of coronary artery disease: Validation, updating, and extension. Eur Heart J 2011;32:1316-30.

10. Genders TS, Steyerberg EW, Hunink MG, et al. Prediction model to estimate presence of coronary artery disease: Retrospective pooled analysis of existing cohorts. BMJ. 2012;344:e3485.

11. Winchester DE, Wolinsky D, Beyth RJ, Shaw LJ. Discordance between appropriate use criteria for nuclear myocardial perfusion imaging from different specialty societies: A potential concern for health policy. JAMA Cardiol 2016;1:207-10.

12. Chaudhuri D, Montgomery A, Gulenchyn K, Mitchell M, Joseph P. Effectiveness of quality improvement interventions at reducing inappropriate cardiac imaging: A systematic review and metaanalysis. Circ Cardiovasc Qual Outcomes 2016;9:7-13.

Publisher's Note Springer Nature remains neutral with regard to jurisdictional claims in published maps and institutional affiliations. 